\title{
Optimal grid pattern model for search and rescue operation in dipterocarp forest research methodology
}

\author{
S. N. Alsagoff \\ Faculty of Science and Defence Technology, \\ National Defence University of Malaysia, Malaysia
}

\begin{abstract}
Many people go lost or missing in dense jungle or forest area each year. When this occurs, time is of the essence when trying to locate the person. If the person is just lost, he or she can try to find the closest trail and follow the trail back. The problem occurs when the person is injured, incapacitated or even dead. It is up to the search and rescue (SAR) teams to locate the person at the search area. The search area is an area where the lost or missing person is most likely to be located. To overcome this problem, a research on optimal grid pattern model for search and rescue operation in dipterocarp forest will be conducted.
\end{abstract}

Keywords: search and rescue, formation, maneuver, GPS, network centric, command and control.

\section{Introduction}

When a SAR mission is conducted, tactics are used to maximize the search capability of the team. It includes the ordered arrangement and manoeuvre of the SAR team members in relation to each other. Command and control are required to ensure that the search area is effectively and efficiently searched.

Currently, all command and control of the arrangement and maneuver of team are conducted through either visual or verbal communication. The SAR team leader will also need to be aware of the location of his SAR team members to ensure that no part of the search area is missed. In a dense jungle or forest environment, with its various thick flora and fauna, command and control will be difficult to maintain. The lack of command and control can even lead to lost 
SAR team members and the missing person not found even if in the close vicinity [1].

This research will focus on the dipterocarp forest which is the biggest type in East Malaysia. A dipterocarp forest is populated by trees from the Dipterocarpaceae family. The dipterocarp forests are found on dry land just above sea level to an altitude of about 900 metres. The lower level of the forest is made up of dense small trees and vegetations [2] as shown in Figure 1.

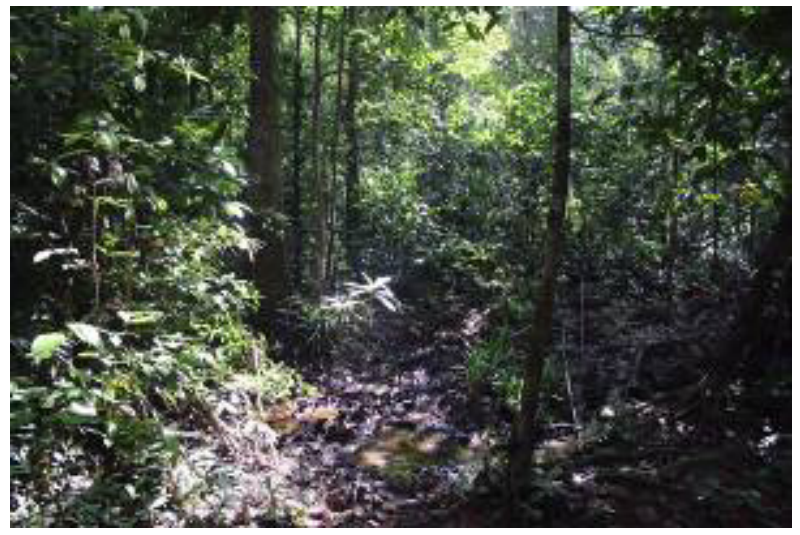

Figure 1: Dipterocarp forest.

\section{Objective}

The objective of this study is to analyse issues related to SAR operations in dipterocarp forest and to propose the methodology to generate optimal grid pattern model for search and rescue operations in dipterocarp forest.

\section{Statement of problem}

\subsection{Search and rescue tactics}

From the National Search And Rescue Manual Volume I: National Search And Rescue System, it is stated that grid patterns are generally the most effective for land search. A grid pattern search allows for the most systematic way of searching an area. The grid search pattern is set on the map depending on the number of the SAR team members and the terrain. A searchline is first formed along the start of the grid pattern at the search area boundary, with individual SAR team members positioned one track spacing apart [3].

After the searchline is formed, it moves forward on a signal from the SAR team leader. The SAR team members remain evenly spaced as the team progresses. If part of the searchline encounters an obstacle, the affected member should investigate it while the rest of the team continues just past the obstacle and stops to wait. When the investigation is complete, the investigators will 
rejoin the searchline and the entire searchline again moves forward on signal of the SAR team leader [3].

\subsection{Environmental factors in SAR efforts}

In dense jungle or forest environment, both visual and vocal contact must be maintained between adjacent SAR team members. This ensures full area coverage and protection for all SAR team members. Whenever contact is lost, the SAR team leader must be aware to avoid lost SAR team members. The searchline will then stop until complete team contact is reestablished. If the searchline is more open, only adjacent visual contact must be maintained, though vocal contact is usually also maintained.

The SAR team leader always makes the final determination of track spacing. Generally, track spacing depends on target characteristics, weather, and terrain. Track spacing for lost or missing persons is typically between 15 and 25 feet [3], with search progress through jungle or forest areas conducted at a slow walking speed, and all dense undergrowth and depression checked. Depending on the undergrowth, the track spacing might be smaller [3].

The SAR team will need to communicate either verbally or visually. Dense undergrowth might hinder the communication among SAR team members. This might lead to the breaking up of the searchline and lessening the effectiveness of the search effort. Once the grid pattern search is finished with no result, other possible areas will be search using the same method.

\section{Conceptual framework}

\subsection{Effective SAR command and control}

Current effectiveness of SAR teams can be enhanced through the use of Network Centric and GPS Technology. Network Centric Technology focuses on the central role of integrated networking and computing of improving interactions among the various entities [4]. GPS Technology provides reliable positioning and navigation services to users which has an unobstructed view of four or more GPS satellites.

\subsection{GPS technology}

The Global Positioning System is a space-based navigation and positioning system that was designed by the US Military to allow a single soldier or group of soldiers to autonomously determine their position to within 10 to 20 meters of truth [5]. Even though developed by the military, GPS technology is now used by everyone to determine their current location and how to get to a place using a GPS receiver.

GPS itself is a distance/ranging system. It operates on the principle of trilateration. In trilateration, the position of an unknown point is determined by measuring the lengths of the sides of a triangle between the unknown point and two or more known points in this case the satellites [5]. Reliable positioning and 
navigation services require four or more GPS satellites because the more satellites are "locked on", the more accurate the timings of the satellites signal reaching the GPS receiver.

\subsection{GPS receiver}

There are many different types of GPS receiver available in the market but this paper will concentrate on the portable handheld GPS receiver. In all cases, the function of a portable handheld GPS receiver will be to determine the location of the user and also to guide the user to a preset location. Indeed the use of the GPS is so widespread, that it is now a must as a standard for higher-end mobile phones. In 2009, 150 million GPS enabled mobile phones were sold [6].

\subsection{GPS tracking}

Currently, GPS receiver is mainly used to determine the location of the user and to guide the use to a preset location. For a SAR team command and control, the SAR team commander must be able to know the location of each of his team members and guide them to a preset location. This means the GPS receiver must be able to receive location coordinates from the commander and also send back its coordinates. All GPS receivers in the market are standalone unit without the means to send and receive data except for the mobile phone. Higher-end GPS enabled mobile phone are also Wi-Fi enabled. Current readily available router technology allows for Wi-Fi coverage to be up to 300 meters outdoors.

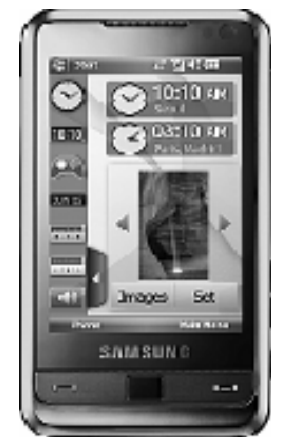

Figure 2: Samsung Omnia phone.

For the purpose of this research, the Samsung Omnia phone as shown in Figure 2 will be used. It runs Window Mobile 6.5 and is GPS and Wi-Fi enabled.

\subsection{GPS effectiveness in forest environment}

As proven in Figure 3 below, even under thick forest canopy, the Samsung Omnia did not lose GPS lock. The red line shows the movement of the Samsung Omnia through forest environment. 


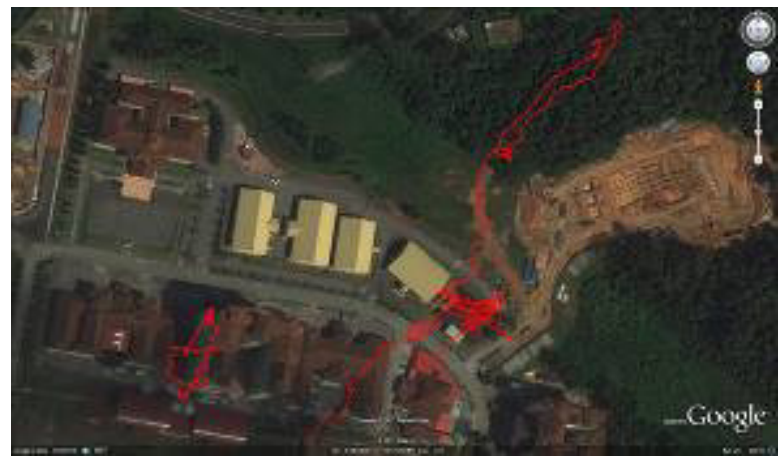

Figure 3: GPS effectiveness in forest environment.

\subsection{System development}

The Samsung Omnia is chosen because it has proven to be a capable and robust Wi-Fi and GPS enabled phone. In addition to that, it is also running on Windows Mobile 6.5. This allows for user to develop and run their own application as compared to running on an IOS devices such as the IPhone (the IOS devices need to be hacked to be able to run applications not authorised by Apple).

The system will be a client-server application. The client side on the Samsung Omnia will be developed using Basic4PPC, a Visual Basic like programming language for Windows Mobile phone. Basic4PPC is based on the Microsoft .Net framework. The advantages of programming with Basic4PPC are its simplicity and the ease to integrate and control other Window Mobile phone hardware component.

The Wi-Fi and GPS component of the Samsung Omnia will be used for the system development. The diagram for the system will be as in Figure 4.

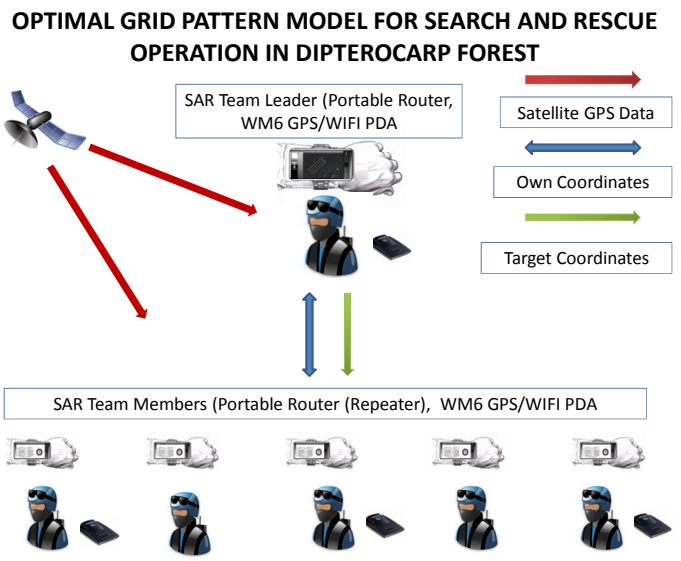

Figure 4: System diagram. 
The server side will be developed using Microsoft Visual Basic 6. Franson GPS Tools will be used to provide the mapping capability for Visual Basic 6 . The map itself will be from Google Maps. The area of operation will be captured and saved as a JPEG file. The JPEG file will first need to be calibrated before it can be used. At least 3 different prominent landmark points that are suitability far enough on the JPEG will be chosen. The coordinates for the prominent landmarks can be retrieved from Google Maps or Google Earth. The coordinates are inputted into GPS Tools toolkit to calibrate the JPEG file. Once done, the JPEG file will be saved as a ".MAPLIB" file that can be loaded into Visual Basic 6.

\subsection{Network centric and GPS technology}

Each of the SAR team will carry a GPS unit based on the Samsung Omnia as shown in Figure 5.

The GPS unit will continuously send back its coordinate to the server. Upon receiving the coordinate, the server will display the location of the SAR team member as an icon on the map. If there are 10 team members on the ground, there will be 10 icons on the screen. The display of the server with 4 SAR team members is shown in Figure 6.

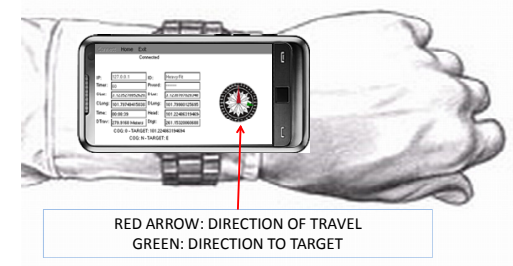

Figure 5: $\quad$ SAR client.

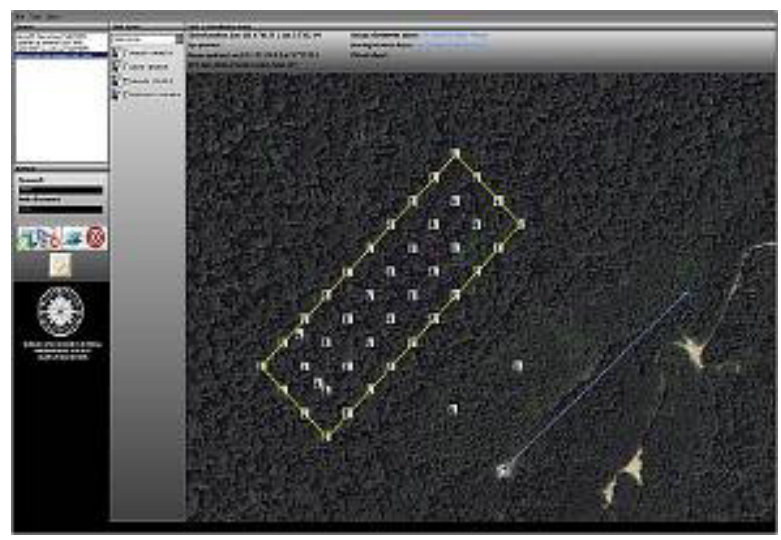

Figure 6: SAR server. 
The data communication between client and server will be continuous. The data flow is shown in Figure 4 above. The server can send target coordinate to SAR team members individually. The capability for the server to send target coordinates individually combined with SAR team member tracking allows for the research methodology to be executed.

\section{Methodology}

\subsection{Research methodology}

The research methodology used will be searching of a test area for a test item. The SAR test team will not know the actual location of the test item. The location of each SAR team member will be tracked using Network Centric and GPS technology as shown in Figure 6. The size and pattern of the search grid and the distance between the SAR team members will be monitored to determine the optimal search grid pattern. If the test item can be found $100 \%$ at all times, then the optimal search grid pattern is found. The results must be at $100 \%$ because human lives are at stake.

\section{2 "Getting there"}

When the SAR team member receives the target coordinate from the server, he will need to know how to get to the target location. For this purpose he will get the distance and the direction to travel. The SAR client will automatically do this for him. Based on the current coordinate and the target coordinate, the SAR client will calculate the direction of travel and distance to travel. The mathematics of the calculation will not be discussed here. The direction to travel display on the SAR Client is shown in Figure 7. The red arrow shows the direction of travel while the green arrow shows the direction to target.

If the SAR team member has to divert from the direction of travel, the SAR client will continuously make the necessary calculation and adjustment to direction to travel and distance to travel to reach the target location.

\section{TARGET INDICATOR}

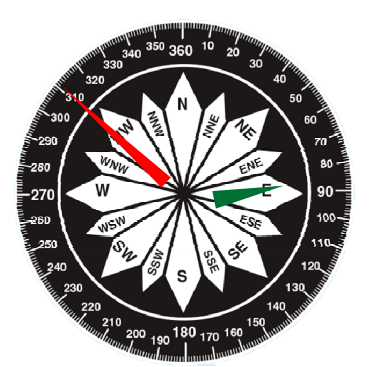

ALIGN BOTH RED AND GREEN ARROWS AND MOVE TOWARD NORTH IN THE INDICATOR TO REACH TARGET

Figure 7: Direction to travel. (See online for colour version.) 


\section{3 "Stopping"}

If the SAR team member follows the given direction to travel, the distance to travel will decrease. Depending on the accuracy setting by the SAR server, the SAR team member will stop about several meters from the target location. He will get the alert signal to stop from a flashing indicator and vibration from the SAR client.

\subsection{Search grid pattern}

The SAR server will need to generate the search grid based on the numbers to SAR team members. The distance between SAR team members will be set based on the thickness and visibility factor of the forest environment. The thicker the forest and the lower the visibility factor, the closer the SAR team members need to be with each other in the parallel search line. The configuration screen for the search grid pattern generator is shown in Figure 8.

The generated search grid pattern will be overlaid on the map as shown in Figure 9.

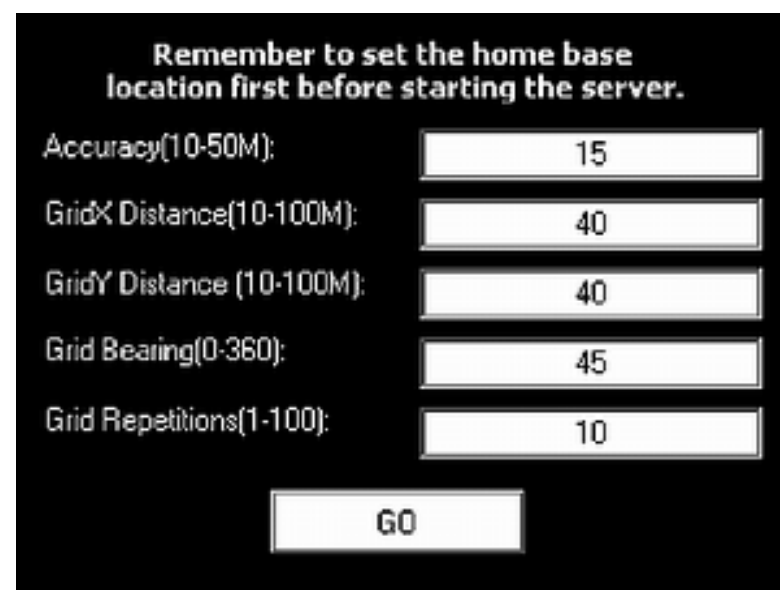

Figure 8: Configuration screen for search grid pattern generator.

\subsection{Moving in parallel line}

Moving in parallel line in a forest environment will be a challenge due to a number of factors. They are:

- Thickness

- Visibility

- Fitness

The last factor plays an important role because not everyone has the same level of fitness. Those with a high level of fitness will move faster through a 


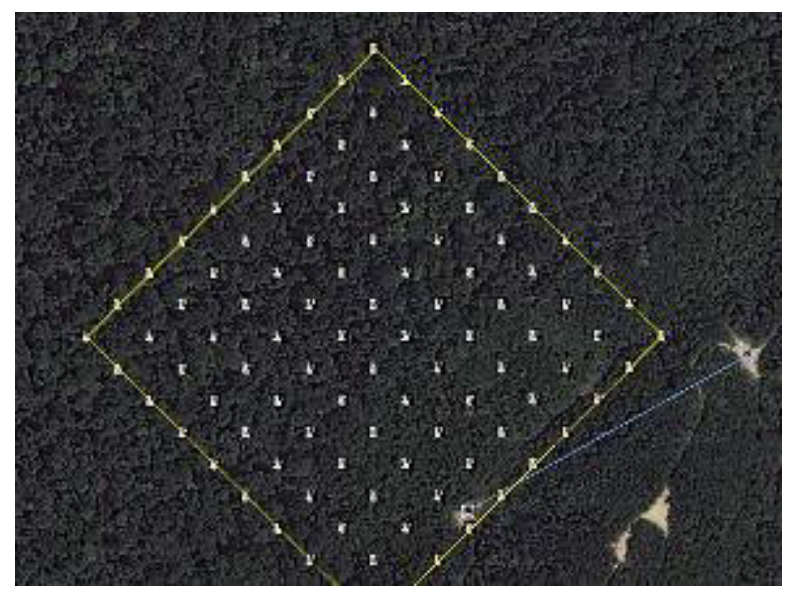

Figure 9: $\quad$ Search grid pattern for 10 SAR team members at $45^{\circ}$ angle.

jungle environment as compared to those who are with lower level of fitness. All the factors will cause the searchline to be staggered thus causing unsystematic search of the area.

To minimize this problem, the SAR server will generate several searchlines parallel to each other. This is shown in Figure 9. When the fastest and fittest SAR team member reaches the first searchline, he will have to wait for the rest of the team to reach the searchline. The SAR server will monitor the rest of the SAR team member. When all of them have reached the searchline, the SAR server will send the command to all SAR clients indicating all team members are to move to the next searchline. This will go on until the search area has been covered

\subsection{Optimal search grid pattern}

Based on the knowledge gained from utilizing the SAR server and client, it is hoped that the optimal search grid pattern can be generated based on the search area forest environment. The SAR team commander will need to input the necessary parametric data to generate the grid pattern as per Figure 8.

\section{Search and rescue tactical management system (SARTAMS)}

At the end of this research, a Search and Rescue Tactical Management System (SARTAMS) will be developed. It will have the following capability:

- $\quad$ SAR team leader can set home base location for all SAR team members to prevent them from getting lost.

- $\quad$ SAR team leader can monitor the location of each SAR team member at all times. 
- $\quad$ SAR team leader can direct SAR team member to different target location individually or by group.

- $\quad$ SARTAMS can generate the optimal search grid for the number of SAR team members and the environment.

- $\quad$ SARTAMS can ensure that all SAR team members in the searchline are always parallel to each other to ensure that all search areas are covered.

- $\quad$ SARTAMS can be adapted for land, sea and air SAR efforts.

- $\quad$ SARTAMS is parametric for large, medium or small search area.

- $\quad$ SARTAMS is parametric for dense or sparse jungle or forest area.

\section{Conclusion}

It is hoped that the Search and Rescue Tactical Management System can assist to locate lost individuals in a forest environment quickly and safely. Time is also an important essence in saving lives. This research project is funded by the Malaysia Ministry of Higher Education Fundamental Research Grant Scheme. Patent has been filed for the Search and Rescue System And Method utilized in SARTAMS.

\section{References}

[1] Ferguson Don, GIS For Wilderness Search And Rescue. Mountaineer Area Rescue Group, ESRI Federal User Conference, February 20-22 2008

[2] Mohd Hasmadi, I., Alias Mohd, S. and Norizah, K. Reclassifying forest type to a new forest class based on vegetation and lithology characteristics using geographic information system at southern Johore, Malaysia. International Journal of Energy and Environment, Issue 4 Volume 2, 2008

[3] U.S Coast Guard, National Search and Rescue Manual Volume I: National Search and Rescue System. Jan 1992

[4] Barabasi Alberto-Laszlo, Linked, The New Science of Networks, How Everything is Connected to Everything Else and What it Means for Science, Business and Everybody Life, Pursues Publishing, 2002

[5] Gregory T. French, Understanding the GPS: An Introduction to the Global Positioning System, GeoResearch, Inc, 1996

[6] Berg Insight, GPS and Mobile Handsets - 4th edition LBS Research Series 